\section{Emotional stress from watching soccer can increase the risk of cardiac events}

The hypothesis that emotional stress, such as that experienced by soccer fans while watching an important match, can increase the risk of cardiac events is controversial. During the Fédération Internationale de Football Association (FIFA) World Cup held in Germany in 2006, Wilbert-Lampen et al. prospectively assessed the incidence of acute cardiac events in the greater Munich area to determine whether the incidence of such emergencies was increased on those days on which the German team played a match (match days).

In comparison with the incidence of cardiac events during the control periods (1 May to 8 June 2006, 10 July to 31 July 2006, and 1 May to 31 July in 2003 and 2005), the incidence of cardiac emergencies on match days was 2.66 times higher $(P<0.001)$; the increase was particularly marked in men. The highest incidence of events was observed during the $2 \mathrm{~h}$ after the start of the match, which is concordant with the emotional stress experienced during the match being the trigger for the event. Notably, the number of cardiac events in patients with known coronary artery disease (CAD) increased by a factor of 4.03 on match days; the corresponding increase in patients without a history of CAD was by a factor of 2.05. A subanalysis of serious events showed significant increases in the incidences of both myocardial infarction without ST-segment elevation and the composite of cardiac arrhythmia causing major symptoms.

These findings indicate that the emotional stress associated with watching a soccer match can increase the risk of acute cardiac events, particularly in men with a history of CAD.

Original article Wilbert-Lampen U et al. (2008) Cardiovascular events during World Cup soccer. $N$ Engl J Med 358: $475-483$

\section{Adverse events cluster after cessation of clopidogrel therapy}

Current cardiology guidelines suggest that clopidogrel therapy should be continued for about 1 year following hospitalization for acute coronary syndromes (ACS). Ho et al. examined the effects of clopidogrel cessation in a retrospective study of 3,137 patients with ACS who had taken clopidogrel as posthospital treatment.

The study cohort ( $98 \%$ male; median age 70 years) included 1,568 patients who had been medically treated, and 1,569 patients who had undergone percutaneous coronary intervention (PCl), at Veterans Affairs hospitals in the US. None of the patients included in the study had experienced an adverse event while taking clopidogrel. Multivariate analyses showed that there was a nearly twofold higher risk of the combined end point of death or acute myocardial infarction in the 90-day period after cessation of clopidogrel treatment than during the period $91-180$ days after cessation (adjusted incidence rate ratios 1.98 [95\% Cl 1.46-2.69] for patients who were medically treated and 1.82 [95\% Cl 1.17-2.83] for patients who underwent $\mathrm{PCl})$. This increased risk remained significant regardless of duration of clopidogrel treatment $(\leq 3, \leq 6, \leq 9$ or $>9$ months) or presence of diabetes mellitus. These findings support the hypothesis that a rebound hyperthrombotic period-as a result of rebound platelet activation-occurs after treatment with clopidogrel is stopped.

The authors state that in patients with ACS, regardless of $\mathrm{PCl}$, the risk upon cessation of clopidogrel therapy must be weighed against the risk of bleeding that is associated with prolonged antiplatelet treatment, and that a change in current guidelines could be warranted.

Original article Ho PM et al. (2008) Incidence of death and acute myocardial infarction associated with stopping clopidogrel after acute coronary syndrome. JAMA 299: 532-539

\section{Thrombus aspiration during PCI improves myocardial reperfusion}

Primary percutaneous coronary intervention $(\mathrm{PCl})$ is the standard treatment for ST-segment elevation myocardial infarction (STEMI). Although $\mathrm{PCl}$ is effective at opening the infarctrelated artery, embolization of plaque or thrombotic material can result in microvascular obstruction. The use of a manual-aspiration catheter during primary $\mathrm{PCl}$ could improve myocardial perfusion. Researchers from the University of Groningen in The Netherlands conducted a randomized trial to determine whether $\mathrm{PCl}$ with thrombus aspiration resulted in better myocardial reperfusion than conventional PCI. 